\title{
Axisymmetric Flow Through a Cylinder with Porous Medium
}

\author{
Muhammad Umar Farooq", Abdul Rehman, Naveed Sheikh, Manzoor Iqbal
}

Department of Mathematics, University of Balochistan, Quetta, Pakistan

Email address:

umarfarooq71965@gmail.com (M. U. Farooq)

*Corresponding author

\section{To cite this article:}

Muhammad Umar Farooq, Abdul Rehman, Naveed Sheikh, Saleem Iqbal. Axisymmetric Flow Through a Cylinder with Porous Medium. International Journal of Systems Science and Applied Mathematics. Vol. 5, No. 3, 2020, pp. 32-35. doi: 10.11648/j.ijssam.20200503.12

Received: October 2, 2020; Accepted: October 20, 2020; Published: October 27, 2020

\begin{abstract}
The present work is a struggle to establish a mathematical appearance of the conduct of axisymmetric fluid flow in a moving cylinder confined in a porous medium. The fluid is assumed to be flowing through the annular region formed between two concentric smooth cylinders for the case when the outer cylinder is kept fixed while the inner cylinder is assumed to be moving with a constant velocity along the axial direction and is also assumed to be rotating with a constant angular velocity with reference to the centre line along the axial axis. Firstly, the conducting equations of motion are obtained in the form of a system of coupled non-linear partial differential equations with corresponding boundary conditions. The system is then transformed into a new set of coupled non-linear ordinary differential equations using a set of suitable similarity transformation. The problem is then solved using the fourth order numerical technique, the Runge-Kutta-Shooting method. The concluding results are derived for non- dimensional coupled differential equations. In the end the results are graphically presented and the behaviour of porosity parameter over the fluid flow is examined. The observed results indicated that with increasing values of the Reynolds's numbers the non-dimensional linear and axial velocities also increases.
\end{abstract}

Keywords: Moving Cylinder, Porous Medium, Runge-Kutta $4^{\text {th }}$ Order Shooting Method

\section{Introduction}

From the last quarter of the previous century, the study of lubricants protecting the moving cylinder in the automobiles as well as in industrial machines had become a research target. Considering the required portion of medicine for a particular disease in a syrup, the solid particles are quite effectively used. Although the petro chemical liquid is area of interest of scientists for a long period to get new substances but the nature of different solid particles present in the fluids changes the results in targeted areas. The stable state viscous glide as well as thermal conductivity of a fluid with density variation connected to temperature change, concludes the result of the exact solution of the Navier-Stokes velocity and energy equation extracted in the case, here temperature of disc or its associated wall heat is treated as fixed. Putra et al. [1] studied the behaviour of a nanofluid depending upon the ingredients composition, correlation and size of the particle. Kuznetsov \& Nield [2] explained the thermal irregularity. Khan and Pop [3] studied the boundary layer flow on an extended covering. Nadeem and Rehman [4] discussed the stagnation-flow of a nanofluid over an axial cylinder. From infinite circular cylinder heat transfer and fluid glide around the surface was studied by Khan et al. [5]. An analytic approach was performed for heat transfer and fluid flow along elliptical cylinders by Khan et al. [6]. Covering Yawed circular cylinders by real fluid flow was presented in mathematical relation considering crosswise and piecewise velocity by Chiu and Lien hard [7]. Around a round cylinder, similarities of flow and heat transfer along drag coefficient idea were floated by Ma and Duan [8]. Few other related works are cited in [9-11].

A stagnation point is a point in a flow field where the local velocity of the fluid is zero. Stagnation points exist at the surface of objects in the flow field [12-15]. The purpose of the present effort is to study the behavior of fluid flowing through the region formed between two concentric cylinders [16-19] through some porous medium. 


\section{Formulation of the Problem}

The aim of present paper is to study the problem of fluid flow through the annular region formed between two concentric cylinders, where the inner cylinder with the radius $\mathrm{R}$ is assumed to be rotating with a constant angular velocity $\Omega$ and is also translating with a constant velocity $W$ along the axial axis, the outer cylinder with radius $b_{0} R$ is assumed fixed while fluid is injected from the top of the outer cylinder towards the inner cylinder with a constant velocity $U$ along radial axis. The obtained governing set of coupled, nonlinear partial differential equations for conservation of mass and momentum is of the form [20-22]

$$
\begin{gathered}
r \frac{\partial w}{\partial z}+\frac{\partial}{\partial r}(r u)=0 \\
u \frac{\partial u}{\partial r}+w \frac{\partial u}{\partial z}-\frac{v^{2}}{r}=-\frac{1}{\rho} \frac{\partial p}{\partial r}+v\left(\frac{\partial^{2} u}{\partial r^{2}}+\frac{1}{r} \frac{\partial u}{\partial r}+\frac{\partial^{2} u}{\partial z^{2}}-\frac{u}{r^{2}}\right)- \\
\frac{v \phi_{p} u}{k_{0}} \\
u \frac{\partial v}{\partial r}+w \frac{\partial v}{\partial z}+\frac{u v}{r}=v\left(\frac{\partial^{2} v}{\partial r^{2}}+\frac{1}{r} \frac{\partial v}{\partial r}+\frac{\partial^{2} v}{\partial z^{2}}-\frac{v}{r^{2}}\right)-\frac{v \phi_{p} v}{k_{0}} \\
u \frac{\partial w}{\partial r}+w \frac{\partial w}{\partial z}=-\frac{1}{\rho} \frac{\partial p}{\partial z}+v\left(\frac{\partial^{2} w}{\partial r^{2}}+\frac{1}{r} \frac{\partial w}{\partial r}+\frac{\partial^{2} w}{\partial z^{2}}\right)-\frac{v \phi_{p} w}{k_{0}}
\end{gathered}
$$

Where $(u, v, w)$ are the velocity components along $(r, \theta, z)$-axes and $\rho$ is the density.

The associated boundary conditions are

$$
\begin{array}{r}
u(R, z)=0 v(R, z)=R \Omega w(R, z)=W(5) \\
u\left(b_{0} R, z\right)=-U v\left(b_{0} R, z\right)=0 w\left(b_{0} R, z\right)=0(6)
\end{array}
$$

Define the transformation [23-24]

$$
\begin{gathered}
u=-\frac{U f(\eta)}{\sqrt{\eta}}, v=\Omega R h(\eta), \\
w=2 U f^{\prime}(\eta) \xi+W g(\eta), \\
\eta=\frac{r^{2}}{R}, \xi=\frac{z}{R},
\end{gathered}
$$

With the help of the above transformations, Equation (1) is identically satisfied and take the following form.

$$
\begin{array}{r}
\eta \frac{d^{4} f}{d \eta^{4}}+2 \frac{d^{3} f}{d \eta^{3}}+\operatorname{Re}\left(f \frac{d^{3} f}{d \eta^{3}}-\frac{d f}{d \eta} \frac{d^{2} f}{d \eta^{2}}\right)-k p \frac{d^{2} f}{d \eta^{2}}=0 \\
\eta \frac{d^{2} g}{d \eta^{2}}+\frac{d g}{d \eta}+\operatorname{Re}\left(f \frac{d g}{d \eta}-g \frac{d f}{d \eta}\right)-k p g=0 \\
4 \eta \frac{d^{2} h}{d \eta^{2}}+4 \frac{d h}{d \eta}-\frac{h}{\eta}+\operatorname{Re}\left(4 f \frac{d h}{d \eta}+\frac{2 f h}{\eta}\right)-k p h=0
\end{array}
$$

Where $k p=\frac{R^{2} \phi_{p}}{4 k_{o}}$ is the porosity parameter, $R e=\frac{U R}{2 v}$ is the cross-flow Reynolds number and $b=b_{0}$.

The boundary conditions in non-dimensional form are

$$
\begin{gathered}
f(1)=0, \frac{d f(1)}{d \eta}=0, f(b)=\sqrt{b}, \frac{d f(b)}{d \eta}=0, \\
g(1)=1, g(b)=0, h(1)=1, h(b)=0, \\
\theta(1)=1, \theta(b)=0, \psi(1)=1, \psi(b)=0,
\end{gathered}
$$

Introduce the following functions

$$
F_{1}=\frac{d f}{d \eta}, F_{2}=\frac{d F_{1}}{d \eta}, F_{3}=\frac{d F_{2}}{d \eta}, G=\frac{d g}{d \eta} \text { and } H=\frac{d h}{d \eta}
$$

Then the set of equations (10-12) and the boundary conditions (13) take the following form

$$
\begin{array}{r}
\eta \frac{d F_{3}}{d \eta}+2 \frac{d F_{2}}{d \eta}+\operatorname{Re}\left(f \frac{d F_{2}}{d \eta}-\frac{d f}{d \eta} \times \frac{d F_{1}}{d \eta}\right)-k p \frac{d^{2} f}{d \eta^{2}}=0 \\
\eta \frac{d G}{d \eta}+\frac{d g}{d \eta}+\operatorname{Re}\left(f \frac{d g}{d \eta}-\frac{d f}{d \eta} g\right)-k p=0 \\
4 \eta \frac{d H}{d \eta}+4 \frac{d h}{d \eta}-\frac{h}{\eta}+\operatorname{Re}\left(4 f \frac{d h}{d \eta}+\frac{2 f h}{\eta}\right)-k p=0
\end{array}
$$

The boundary condition in non-dimensional form are

$$
\begin{aligned}
& f(1)=0, F_{1}(1)=0, f(b)=\sqrt{b}, F_{1}(b)=0, \\
& g(1)=0, g(b)=0, h(1)=1, h(b)=0 .
\end{aligned}
$$

\section{Results and Discussion}

The presented research paper is an effort to provide a numerical pattern that observes the flow along the surface of a rotating as well as vertically moving cylinder in a porous medium. The numerical results are calculated for nondimensional system of first order differential equations by using the $4^{\text {th }}$ order Runge-Kutta shooting method [25-28]. In Figure 1 it is observed that the velocity profile increases. Figure 1 shows the behaviour of velocity profile for different values of Reynolds Number Re. Figure 2 shows the behaviour of velocity gradient for different values of the Reynolds Number Re. From figure a dual behaviour is observed. In Figure 3 the non-dimensional velocity $g$ decreased for increasing values of the porosity parameter $k p$. Figure 3 shows the behaviour of velocity profile $g$ for different values of the porosity parameter $k p$. Figure 4 shows the behaviour of velocity profile $h$ for different values of the porosity parameter $k p$. From the figure it is observed that with increase in the non-dimensional velocity profile $h$ increases for increasing values of the porosity parameter $k p$.

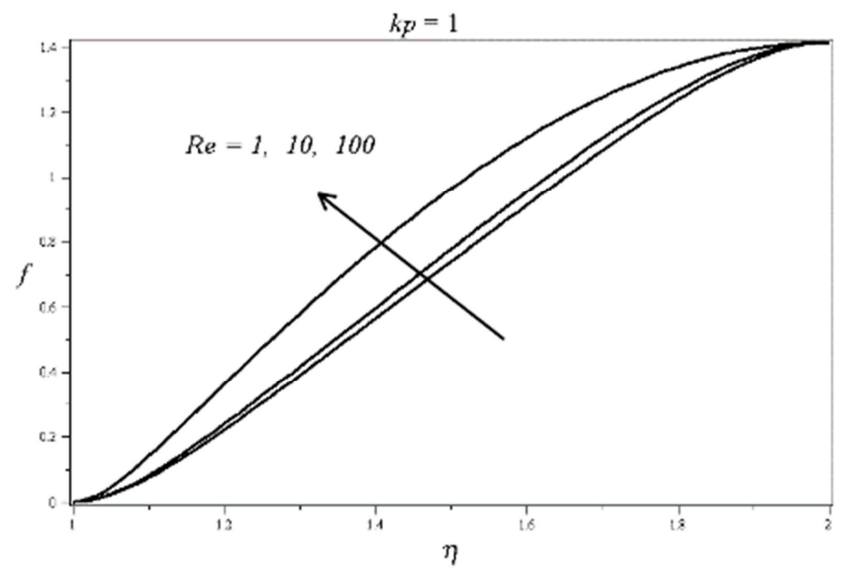

Figure 1. Behaviour of non-dimensional velocity profile ffor different values of the Reynolds number Re. 


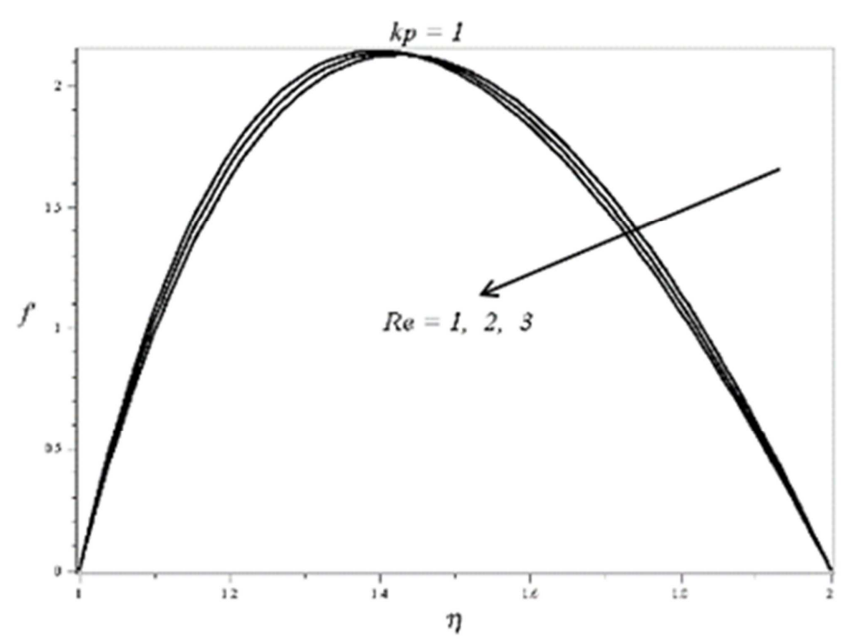

Figure 2. Behaviour of non-dimensional velocity gradient $f^{\prime}$ for different values of the Reynolds number Re.

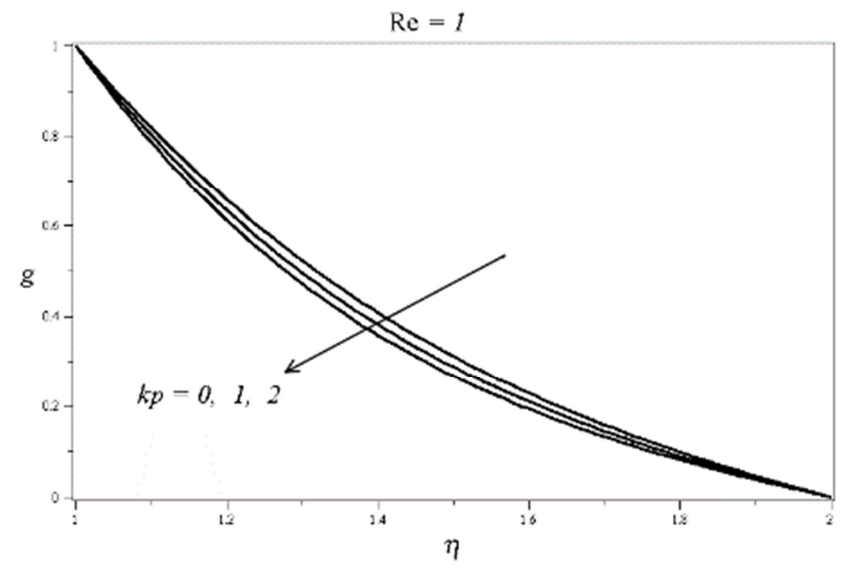

Figure 3. Behaviour of non-dimensional velocity $g$ for different values of the porosity parameter $k p$.

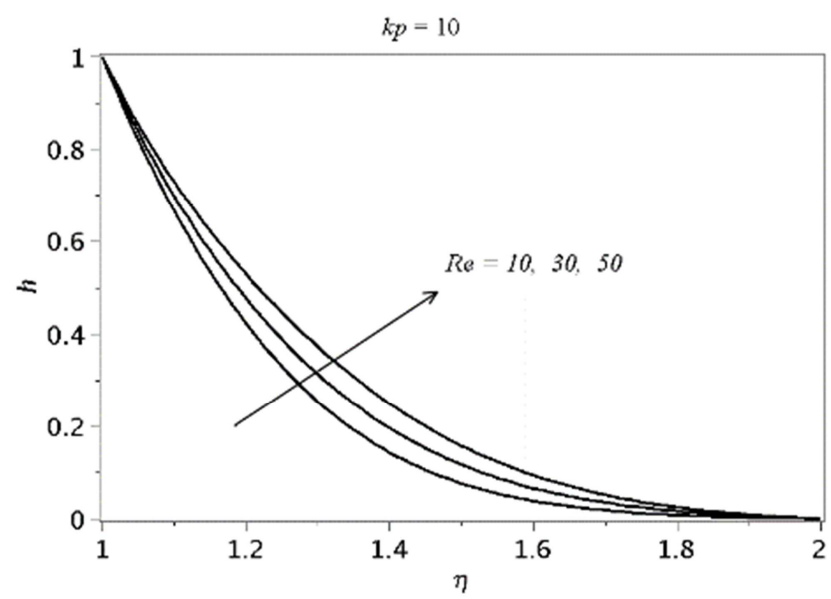

Figure 4. Behaviour of non-dimensional velocity $h$ for different values of the porosity parameter $\mathrm{kp}$.

\section{Conclusion}

Main conclusions from the above analysis are:

With increase in the Reynolds numbers the nondimensional velocity profile $f$ increases.
With increase in the porosity parameter the nondimensional velocity profile $g$ decreases.

With increase in the Reynolds numbers the nondimensional velocity profile $h$ increases.

\section{References}

[1] N. Putra, W. Roetzel, S. K. Das, Natural convection of nanofluids. Heat and mass transfer, 39 (8-9), (2003), 775-784.

[2] D. A. Nield, A. V. Kuznetsov, Thermal instability in a porous medium layer saturated by a nanofluid. International Journal of Heat and Mass Transfer, 52 (25-26), (2009), 5796-5801.

[3] W. A. Khan, I. Pop, Boundary layer flow past a wedge moving in a nanofluid. Mathematical Problems in Engineering, (2013).

[4] Nadeem, S., \& Rehman, A. (2013). Axisymmetric stagnation flow of a nanofluid in a moving cylinder. Computational mathematics and modeling, 24 (2), 293-306.

[5] Khan, W. A., J. R. Culham, and M. M. Yovanovich. "Fluid flow around and heat transfer from an infinite circular cylinder." (2005), 785-790.

[6] W. A. Khan, J. R. Culham, M. M. Yovanovich, Fluid flow around and heat transfer from elliptical cylinders: analytical approach. Journal of thermophysics and heat transfer, 19 (2), (2005), 178-185.

[7] W. S. Chiu, J. H. Lienhard, On real fluid flow over yawed circular cylinders (1967).

[8] H. Ma, Z. Duan, Similarities of Flow and Heat Transfer around a Circular Cylinder. Symmetry, 12 (4), (2020), 658.

[9] S. Nadeem, Abdul Rehman, Mohamed Ali, The boundary layer flow and heat transfer of a nanofluid over a vertical slender cylinder, J. NanoEngineering and NanoSystems (2012) 1-9.

[10] S. Nadeem, Abdul Rehman, Changhoon Lee, Jinho Lee, Boundary layer flow of second grade fluid in a cylinder with heat transfer, Mathematical Problems in Engineering, Volume 2012, Article ID 640289.

[11] S. Nadeem, Abdul Rehman, K. Vajravelu, Jinho Lee, Changhoon Lee, Axisymmetric stagnation flow of a micropolar nanofluid in a moving cylinder, Mathematical Problems in Engineering, Volume 2012, Article ID 378259.

[12] Abdul Rehman, S. Nadeem, Mixed convection heat transfer in micropolar nanofluid over a vertical slender cylinder, Chin. Phy. Lett. 29 (12) (2012) 124701-5.

[13] S. Nadeem, Abdul Rehman, Axisymmetric stagnation flow of a nanofluid in a moving cylinder, Comp. Math. Mod. 24 (2) (2013) 293-306.

[14] Abdul Rehman, S. Nadeem, M. Y. Malik, Stagnation flow of couple stress nanofluid over an exponentially stretching sheet through a porous medium, J. Power Tech. 93 (2) (2013) 122132 .

[15] Abdul Rehman, S. Nadeem, M. Y. Malik, Boundary layer stagnation-point flow of a third grade fluid over an exponentially stretching sheet, Braz. J. Che. Eng. 30 (3) (2013) 611-618. 
[16] Abdul Rehman, S. Nadeem, Heat transfer analysis of the boundary layer flow over a vertical exponentially stretching cylinder, Global J. Sci. Fron. Res. 13 (11) (2013) 73-85.

[17] M. Y. Malik, M. Naseer, S. Nadeem, Abdul Rehman, The boundary layer flow of Casson nanofluid over a vertical exponentially stretching cylinder, Appl. NanoSci. DOI: 10.1007/s13204-012-0267-0.

[18] Abdul Rehman, S. Nadeem, S. Iqbal, M. Y. Malik, M. Naseer, Nanoparticle effect over the boundary layer flow over an exponentially stretching cylinder, J. NanoEngineering and NanoSystems (2014) 1-6.

[19] M. Y. Malik, M. Naseer, S. Nadeem, Abdul Rehman, The boundary layer flow of hyperbolic tangent fluid over a vertical exponentially stretching cylinder, Alexandria Eng. J., 53 (2014) 747-750.

[20] Abdul Rehman, R. Bazai, S. Achakzai, S. Iqbal, M. Naseer, Boundary Layer Flow and Heat Transfer of Micropolar Fluid over a Vertical Exponentially Stretched Cylinder, App Comp Math, 4 (6) (2015) 424-430.

[21] Abdul Rehman, G. Farooq, I. Ahmed, M. Naseer, M. Zulfiqar, Boundary Layer Stagnation-Point Flow of Second Grade Fluid over an Exponentially Stretching Sheet, American J App Math Stat, 3 (6) (2015) 211-219.

[22] Abdul Rehmana, S. Achakzai, S. Nadeem, S. Iqbal, Stagnation point flow of Eyring Powell fluid in a vertical cylinder with heat transfer, Journal of Power Technologies 96 (1) (2016) $57-62$.
[23] Abdul Rehman, Saleem Iqbal, Syed Mohsin Raza, Axisymmetric Stagnation Flow of a Micropolar Fluid in a Moving Cylinder: An Analytical Solution, Fluid Mechanics, 2 (1) (2016) 1-7.

[24] Naheeda Iftikhar, Abdul Rehman, Peristaltic flow of an Eyring Prandtl fluid in a diverging tube with heat and mass transfer, International Journal of Heat and Mass Transfer 111 (2017) $667-676$.

[25] Abdul Rehman, Naveed Sheikh, Boundary Layer StagnationPoint Flow of Micropolar Fluid over an Exponentially Stretching Sheet, International Journal of Fluid Mechanics \& Thermal Sciences, 2017; 3 (3): 25-31.

[26] Haroon Rasheed, Abdul Rehman, Naveed Sheikh, Saleem Iqbal, MHD Boundary Layer Flow of Nanofluid over a Continuously Moving Stretching Surface, Applied and Computational Mathematics, 2017; 6 (6): 265-270.

[27] Najeeb Alam Khan, Umair Bin Saeed, Faqiha Sultan, Saif Ullah, and Abdul Rehman, Study of velocity and temperature distributions in boundary layer flow of fourth grade fluid over an exponential stretching sheet, AIP ADVANCES 8, 025011 (2018).

[28] Naheeda Iftikhar, Abdul Rehman, Hina Sadaf, Muhammad Najam Khan, Impact of wall properties on the peristaltic flow of $\mathrm{Cu}$-water nano fluid in a non-uniform inclined tube, International Journal of Heat and Mass Transfer 125 (2018) 772-779. 